\title{
AVALIAČ̃̃ DE DIFERENTES DIETAS SOBRE O DESEMPENHO DE BEZERROS DA RAÇA HOLANDESA DURANTE O PERÍODO DE ALEITAMENTO
}

\author{
Effect of differents diets on the performance of holstein calves during suckling period
}

\author{
Paula Alves Teixeira ${ }^{1}$, Mauro Dal Secco de Oliveira ${ }^{2}$, Clayson Correia de Sousa ${ }^{3}$, \\ Tiago Máximo da Silva ${ }^{4}$
}

\begin{abstract}
RESUMO
Foram utilizados 24 bezerros da raça Holandesa distribuídos em delineamento inteiramente casualizado com oito repetições e três tratamentos: leite integral (LI) e dois tipos de sucedâneos, DestetorÒ (DES) e LactalÒ (LAC), durante o período de aleitamento (60 dias), sendo avaliados: ganho de peso, consumo de matéria seca e mensurações corporais (altura na cernelha, circunferência toráxica e ventral). Os ganhos médios diários de peso, a altura na cernelha e a circunferência torácica e ventral dos bezerros não diferiram $(\mathrm{P}>0,05)$ entre os tratamentos. Foram obtidas as médias de ganho de peso de $0,32,0,41$ e $0,26 \mathrm{~kg} / \mathrm{bezerro} / \mathrm{dia}$ nos tratamentos LI, DES e LAC, respectivamente. O consumo de matéria seca total na fase de aleitamento, durante 60 dias, diferiu entre os tratamentos, sendo que os bezerros que receberam o sucedâneo DES consumiram mais matéria seca total, comparados àqueles que receberam o sucedâneo LAC $(\mathrm{P}<0,05)$. As médias de consumo diário de matéria seca foram: 0,$79 ; 0,94$ e $0,68 \mathrm{~kg} /$ bezerro respectivamente para os tratamentos LI, DES e LAC.
\end{abstract}

Termos para indexação: Consumo, ganho de peso, mensurações corporais, sucedâneo.

\section{ABSTRACT}

24 holstein calves were assigned on a completely randomized experiment with eight repetitions and three treatments: integral milk (LI) and two types of milk replacer, DestetorÒ (DES) and LactalŌ (LAC), during the period of suckling (60 days), when the variables were studied: body weight gain, dry matter intake and corporal measure (wither height, heart and abdominal girth circumference). The mean daily body weight gain, wither height, heart and abdominal girth circumference did not had statistically significant differences $(\mathrm{P}>0.05)$. The mean daily body weight gain was $0.32 ; 0.41$ and $0.26 \mathrm{~kg} / \mathrm{calve} / \mathrm{day}$ in LI, DES and LAC treatments respectively. The total dry matter intake was more to the calves that received the DES compared with those that received LAC $(\mathrm{P}<0.05)$. The total daily dry matter intake was: $0.79,0.94$ and $0.68 \mathrm{~kg} /$ calf by the treatments LI, DES and LAC respectively

Index terms: Intake, body weight gain, corporal measures, milk replacer.

\section{(Recebido em 5 de dezembro de 2006 e aprovado em 10 de abril de 2007)}

\section{INTRODUÇÃo}

Uma das principais vantagens do aleitamento artificial é a possibilidade de controlar o consumo de leite pelos animais e promover o desaleitamento precoce, já que o aleitamento natural tem um custo muito elevado (LOMBARDI et al., 1997).

A redução na idade do desaleitamento, com a diminuição da quantidade de leite integral fornecido aos bezerros ou a substituição por sucedâneo de custo inferior, reduziria o custo da alimentação, além de possibilitar ao produtor de leite o envio de maiores quantidades de leite para o consumo humano. Contudo, o fornecimento de sucedâneo ao bezerro durante o período de aleitamento não deve deixar de possibilitar tanto por meio da dieta líquida quanto da sólida uma nutrição adequada, a fim de promover um desempenho que mantenha os animais numa condição corporal compatível com a definição para os padrões da raça. Nesse contexto, além da disponibilidade do volumoso, o fornecimento do concentrado nutricionalmente adequado

\footnotetext{
Zootecnista, Mestre - Departamento de Zootecnia/DZ - Faculdade de Ciências Agrárias e Veterinárias/FCAV - Universidade Estadual Paulista/UNESP _ Via de Acesso Professor Paulo Donato Castellane, s/n - 14884-900 - Jaboticabal, SP - paulinhateixeira@yahoo.com.br

'Zootecnista, Livre Docente, Professor Adjunto - Departamento de Zootecnia/DZ - Faculdade de Ciências Agrárias e Veterinárias/FCAV - Universidade Estadual Paulista/UNESP - Via de Acesso Professor Paulo Donato Castellane, s/n - 14884-900 - Jaboticabal, SP - mauro@fcav.unesp.br Pesquisador do CNPq

${ }^{3}$ Engenheiro Agrônomo, Mestrando em Zootecnia - Departamento de Zootecnia/DZ - Faculdade de Ciências Agrárias e Veterinárias/FCAV - Universidade Estadual Paulista/UNESP - Via de Acesso Professor Paulo Donato Castellane, s/n - 14884-900 - Jaboticabal, SP- Bolsista da CAPES claysoncorreia@yahoo.com.br

${ }^{4}$ Engenheiro Agrônomo, Mestre - Departamento de Zootecnia/DZ - Faculdade de Ciências Agrárias e Veterinárias/FCAV - Universidade Estadua Paulista/UNESP - Via de Acesso Professor Paulo Donato Castellane, s/n - 14884-900 - Jaboticabal, SP-Bolsista do CNPq - tiagounesp@hotmail.com Experimento realizado com auxílio financeiro da FAPESP
} 
e palatável, proporcionará o sucesso do desaleitamento precoce. A dieta sólida é importante, pois permitirá que o bezerro se torne ruminante mais rapidamente, resultando em menor mortalidade e custo (JAURIS et al., 1999; OLIVEIRA, 2001; SUSIN et al., 1988).

Os níveis de substituição do leite integral em sistemas de aleitamento estão intimamente relacionados ao custo comparado do produto substituinte, como sucedâneos à base de soja e soro de queijo (BARRETO, 1993; GERMANO, 1992; LOPES et al., 1998). No entanto, a utilização de produtos desta natureza demonstra, em muitos casos, disparidades no desempenho dos animais, principalmente associados com alimentos de menor aproveitamento, gerando diminuição no ganho de peso e elevação do gasto com medicamentos, quando comparados ao leite integral (VASCONCELOS, 1996). Estes problemas parecem estar relacionados ao excesso de fibra e amido, ao tipo e à inadequada incorporação de gordura além da utilização de fontes protéicas de baixo aproveitamento ou que provocam transtornos digestivos nos bezerros (CAMPOS \& LIZIEIRE, 1995). Aliado a estes aspectos devese atentar para a homogeneidade da mistura final e para a quantidade fornecida aos bezerros, principalmente nas duas semanas iniciais de aleitamento (OLIVEIRA, 2001).
O presente trabalho foi realizado com objetivo de estudar o efeito de diferentes dietas contendo leite integral ou sucedâneo, sobre o desempenho de bezerros da raça holandesa, durante o período de aleitamento.

\section{MATERIAL E MÉTODOS}

O experimento foi conduzido no setor de Bovinocultura de leite da Faculdade de Ciências Agrárias e Veterinárias (FCAV), Campus de Jaboticabal - SP. Foram utilizados 24 bezerros inteiros da raça Holandesa, com cinco dias de idade.

A composição em matéria seca e em nutrientes digestíveis totais do leite foi de 13 e $16 \%$, respectivamente (OLIVEIRA, 2001) e a dos sucedâneos foi a seguinte: Lactal ${ }^{5}=$ soro de leite, lactose, leite desnatado em pó, gordura vegetal homogeneizada, concentrado protéico de soja, acidificante, mistura vitamínico-mineral e coccidiostático (decoquinate); Destetor ${ }^{6}{ }^{6}=$ leite em pó integral ou desnatado, amido de milho pré-gelatinizado, levedura desidratada, farinha de vísceras de aves ${ }^{7}$, óleo vegetal hidrogenado ou gordura animal estabilizada, minerais, aminoácidos, antibióticos, palatabilizantes e mistura vitamínico-micromineral. A identificação dos tratamentos, conforme os tipos de sucedâneos, as quantidades, os períodos de fornecimento e os níveis de garantia dos sucedâneos são apresentados nas Tabelas 1 e 2 .

Tabela 1 - Tipos de sucedâneos, quantidade diária (litros/bezerro) e períodos de fornecimento, conforme os diferentes tratamentos.

\begin{tabular}{|c|c|c|c|c|c|c|}
\hline \multirow{3}{*}{ Períodos } & \multicolumn{6}{|c|}{ Tratamentos } \\
\hline & \multicolumn{2}{|c|}{ Lactal } & \multicolumn{2}{|c|}{ Destetor } & \multicolumn{2}{|c|}{ Leite Integral } \\
\hline & Manhã & Tarde & Manhã & Tarde & Manhã & Tarde \\
\hline 6-14 dias & 2,0 & 2,0 & 2,0 & 2,0 & 2,0 & 2,0 \\
\hline 15-42 dias & 2,0 & 2,5 & 2,5 & 2,5 & 2,0 & 2,0 \\
\hline 43-60 dias & 2,0 & 2,0 & 2,5 & 2,5 & 2,0 & 2,0 \\
\hline
\end{tabular}

*Diluição: Lactal $=100$ gramas/litro de água morna. **Diluição: Destetor $=125$ gramas/litro de água à temperatura ambiente.

Tabela 2 - Níveis de garantia dos sucedâneos.

\begin{tabular}{|c|c|c|c|c|c|c|c|}
\hline \multirow[b]{3}{*}{ sucedâneo } & \multicolumn{7}{|c|}{ Níveis de garantia \% } \\
\hline & $\mathrm{U}$ & PB & $\mathrm{MF}$ & $\mathrm{EE}$ & MM & $\mathrm{Ca}$ & $\mathrm{P}$ \\
\hline & Max. & Min. & Máx. & Mín. & Máx. & Máx. & Mín. \\
\hline LACTAL® & 8,0 & 20,0 & 0,7 & 15,0 & 12,0 & 0,8 & 0,6 \\
\hline DESTETOR $®$ & 10,0 & 22,0 & - & 8,0 & 15,0 & 1,5 & 0,7 \\
\hline
\end{tabular}

$\mathrm{U}=$ Umidade; $\mathrm{PB}=$ Proteína Bruta; MF = Matéria Fibrosa; EE = Extrato Etéreo; MM = Matéria Mineral; Ca = Cálcio; $\mathrm{P}=$ Fósforo. Máx. = Máximo. Mín. = Mínimo.

\footnotetext{
${ }^{5}$ Fabricante: NUTRON.

${ }^{6}$ Fabricante: NUTRIMIX

${ }^{7}$ Embora o MAPA proíba o uso deste ingrediente na alimentação de ruminantes, a empresa possui registro e licença do ministério para a comercialização do produto.
}

Ciênc. agrotec., Lavras, v. 31, n. 6, p. 1831-1837, nov./dez., 2007 
Utilizaram-se abrigos individuais dotados de comedouro, bebedouro e cocho para feno de capim-Tifton 85. Os bezerros receberam colostro (primeiro leite secretado após o parto e impróprio para consumo humano, porém indispensável para conferir imunidade infecciosa aos bezerros) durante os primeiros cinco dias, sendo em seguida submetidos às dietas experimentais (leite ou sucedâneo + concentrado + feno). O concentrado foi composto por milho grão moído, farelo de soja, farelo de trigo e mistura mineral (composição/kg do produto comercial: $\mathrm{P}=73 \mathrm{~g} ; \mathrm{Ca}=190 \mathrm{~g}$; $\mathrm{Na}=62 \mathrm{~g} ; \mathrm{Cl}=90 \mathrm{~g} ; \mathrm{Mg}=44 \mathrm{~g} ; \mathrm{S}=30 \mathrm{~g} ; \mathrm{Cu}=340 \mathrm{mg} ; \mathrm{Mn}=940 \mathrm{mg}$; $\mathrm{Fe}=1064 \mathrm{mg} ; \mathrm{Co}=3 \mathrm{mg} ; \mathrm{I}=16 \mathrm{mg} ; \mathrm{Se}=10 \mathrm{mg} ; \mathrm{F}=730 \mathrm{mg}$ (máximo); veículo qsp=1000g; vitamina $\mathrm{A}=100000 \mathrm{UI}$; D3= 40000UI; E= 600UI.). A composição bromatológica do feno e do concentrado está apresentada na Tabela 3. As análises foram realizadas no Laboratório de Ruminantes/FCAV.

As pesagens, cujo início se deu após o fim do período de fornecimento do colostro e início do aleitamento artificial ( $6^{\circ}$ dia, tomado como dia zero), foram efetuadas pela manhã (antes da refeição) no $29^{\circ}$ no $57^{\circ}$ e no $61^{\circ}$ dia, em balança individual tipo brete. $\mathrm{O}$ consumo tanto do feno quanto do concentrado, em matéria natural, foi medido por diferença na pesagem entre o total fornecido e a sobra no cocho enquanto que, para o sucedâneo ou para o leite não houve sobras, ou seja, o consumo correspondeu à quantidade fornecida aos animais. Por sua vez o consumo de matéria seca (CMS) do concentrado, do feno de capimtifton 85 , da ração (concentrado + feno) e da dieta total (leite + ração) foi calculado com base nos teores de MS apresentados anteriormente na tabela 3. As análises estatísticas dos parâmetros de desempenho, peso e mensurações corporais, foram feitas para cada subperíodo (I, 0 a 28 dias; II, 29 a 56 dias) e para o período total (III, 0 a 60 dias). Estes períodos são diferentes daqueles períodos de fornecimento dos sucedâneos e do leite expressos na tabela 1, os quais apenas atendem à literatura no caso do leite (LUCCI, 1989; OLIVEIRA, 2001) ou às recomendações dos fabricantes, no caso dos sucedâneos, de se realizar o desaleitamento aos 60 dias. Com esta avaliação em tais subperíodos (I, II e III) pretendeu-se subsidiar a discussão deste trabalho quanto ao cumprimento das recomendações dos fabricantes. As medidas da altura na cernelha, circunferência torácica e ventral (média de duas leituras), foram feitas pela manhã (antes da refeição) e após o período de fornecimento de colostro. A primeira mensuração coincidiu com o início do aleitamento (dia zero) e as demais foram realizadas no $29^{\circ}$, $43^{\circ}$ e e no $61^{\text {o- }}$ dia do período experimental.

Utilizou-se o delineamento inteiramente casualizado com 3 tratamentos e oito repetições. A análise de cada variável seguiu o modelo matemático: $X_{i j}=\mu+t_{i}+e_{i j}$, em que $X_{i j}=$ valor da variável na parcela que recebeu o tratamento $i(\mathrm{i}=1$, $2,3)$ na repetição $j(\mathrm{j}=1,2,3,4,5,6,7,8) ; \mu=$ média geral da variável; $t_{i}=$ efeito devido ao tratamento $i(\mathrm{i}=1,2,3)$ que foi aplicado na parcela, e $e_{i j}=$ efeito devido ao acaso na parcela que recebeu o tratamento $i(\mathrm{i}=1,2,3)$ na repetição $j(\mathrm{j}=1,2,3$, $4,5,6,7,8)$. As médias dos tratamentos foram comparadas pelo teste Tukey (BANZATTO \& KRONKA, 1992) a 5 e $1 \%$ de probabilidade, utilizando-se o programa ESTAT, versão 2.0, desenvolvido no Departamento de Ciências Exatas, FCAV, Unesp, Jaboticabal por Barbosa et al. (1992). As médias de ganho de peso foram submetidas à análise de covariância para ajuste do peso inicial dos bezerros.

\section{RESULTADOS E DISCUSSÃO}

As médias de ganho diário de peso dos bezerros nos períodos estudados nos diferentes tratamentos, os valores do teste $\mathrm{F}$ e o erro padrão da média estão expressos na Tabela 4.

Tabela 3 - Composição bromatológica do feno de capim-Tifton 85, do concentrado e do leite integral fornecido para os bezerros durante o período de aleitamento.

\begin{tabular}{cccc}
\hline & Feno de capim Tiftonn & Concentrado & Leite Integral \\
\hline MS (\%) & 91,80 & 90,16 & $13,00 *$ \\
PB (\% da MS) & 3,43 & 18,10 & 3,50 \\
EE (\% da MS) & 0,87 & 3,16 & - \\
FDN (\% da MS) & 77,18 & 19,59 & - \\
MM (\% da MS) & 4,29 & 6,74 & 16,00 \\
NDT $(\%)^{+}$ & 44,61 & 80,63 & - \\
\hline
\end{tabular}

+ Estimativa a partir de Mcdowell et al. (1974). * Segundo Oliveira (2001). MS = Matéria Seca; PB = Proteína Bruta; EE = Extrato Etéreo; FDN = Fibra em Detergente Neutro; FDA = Fibra em Detergente Ácido; MM = Matéria Mineral; NDT = Nutrientes Digestíveis Totais. 
Tabela 4 - Médias de ganho diário de peso (kg/animal) dos bezerros dos diferentes tratamentos e nos diferentes períodos, valores de $\mathrm{F}$ e erro padrão da média (EPM).

\begin{tabular}{ccccc}
\hline & & & Períodos & \\
\cline { 2 - 3 } Tratamentos & I $(0 \mathrm{a} 28$ dias $)$ & II $(29 \mathrm{a} 56$ dias $)$ & & III $(0 \mathrm{a} 60$ dias $)$ \\
LI & 0,22 & $0,41 \mathrm{a}$ & 0,30 \\
DES & 0,14 & $0,65 \mathrm{~b}$ & 0,40 \\
LAC & $-0,01$ & $0,47 \mathrm{a}$ & 0,25 \\
Teste F & $2,28 \mathrm{~ns}$ & $7,46 * *$ & $2,59 \mathrm{~ns}$ \\
EPM & 0,2247 & 0,1278 & 0,1365 \\
\hline
\end{tabular}

Médias seguidas de letras iguais na coluna, não diferem entre si, pelo teste de Tukey. $\mathrm{LI}=$ dieta contendo leite integral. $\mathrm{DES}=$ dieta contendo sucedâneo Destetor. $\mathrm{LAC}=$ dieta contendo sucedâneo Lactal. $\mathrm{ns}=$ não significativo $(\mathrm{P}>0,05) . * *=(\mathrm{P}<0,01)$.

Nos primeiros 28 dias de aleitamento, o ganho diário de peso dos animais foi semelhante $(\mathrm{P}>0,05)$ entre os tratamentos (Tabela 4). A semelhança estatística se deve, provavelmente, ao coeficiente de variação muito elevado $(189,27 ; 24,61$ e 42,32\%, respectivamente nos períodos I, II e II), indicando oscilações acentuadas no ganho de peso dos bezerros. No tratamento LAC os animais perderam peso constantemente, embora estatisticamente não tenha ocorrido diferença. Ficou evidenciado que a queda de peso no período I para o tratamento LAC está relacionada com a maior dificuldade dos bezerros em aproveitarem nutricionalmente o sucedâneo, devido à proteína vegetal (concentrado protéico de soja) utilizado como ingrediente neste sucedâneo. No sucedâneo DES tal ingrediente não é utilizado na formulação.

Analisando-se o período total (0 a 60 dias) de aleitamento, observou-se melhor desempenho dos bezerros pertencentes ao tratamento DES (média de 0,40 $\mathrm{kg} /$ bezerro/dia), embora as médias não tenham diferido entre tratamentos $(\mathrm{P}>0,05)$. $\mathrm{O}$ ganho médio diário dos bezerros do tratamento LI foi de $0,30 \mathrm{~kg} /$ bezerro e dos bezerros do tratamento LAC foi de $0,25 \mathrm{~kg} /$ bezerro. Tudo indica que no tratamento LAC os bezerros se adaptaram mais lentamente ao sucedâneo, possivelmente devido ao menor aproveitamento dos componentes do sucedâneo pelos animais. Plaza \& Fernandez (1997) não encontraram diferença $(\mathrm{P}>0,05)$ no ganho de peso dos bezerros aleitados durante 70 dias, no entanto, o aleitamento com sucedâneo foi mais econômico em relação ao leite integral. Com relação ao ganho de peso médio diário, no período total, a variação de 0,25 a $0,40 \mathrm{~kg} /$ bezerro é condizente com o desempenho esperado dos animais neste período, uma vez que o ganho preconizado pelo NRC (1989) situase entre 0,20 a $0,40 \mathrm{~kg} /$ bezerro/dia. A média de $0,34 \mathrm{~kg} /$ bezerro/dia observada por Sandi \& Mühlbach (1999) em bezerros da raça holandesa desaleitados aos 56 dias de idade (4 litros de leite integral/bezerro/dia) foi muito próxima a obtida no presente estudo $(0,30 \mathrm{~kg} / \mathrm{bezerro} /$ dia). O mesmo ocorreu no trabalho de Jauris et al. (1999), porém com desaleitamento aos 50 dias de idade. Mesmo restringindo a quantidade de leite integral (4 litros/ bezerro/dia até 30 dias e 2,0 litros/bezerro/dia de 31 a 60 dias), Zanetti et al. (1999) obtiveram média de ganho de peso diário de $0,31 \mathrm{~kg} /$ bezerro.

$\mathrm{Na}$ Tabela 5 estão expressas as médias de CMS do concentrado, do feno, da ração e da dieta total dos bezerros nos diferentes tratamentos e períodos, os valores de F, o erro padrão da média e os coeficientes de variação.

Pode-se notar que o consumo de matéria seca, pelos bezerros, do concentrado, do feno de capim-tifton 85 não diferiu entre os tratamentos $(\mathrm{P}>0,05)$, independente do período estudado. Contudo, em relação ao consumo de matéria seca total (CMST), notou-se que em todos os períodos houve diferença $(\mathrm{P}<0,01)$ entre os tratamentos. No PI, os bezerros dos tratamentos LI e DES consumiram mais matéria seca total do que os bezerros do tratamento LAC. A superioridade no CMST dos bezerros dos tratamentos LI e DES foi de $31,91 \%$ em relação ao dos bezerros do tratamento LAC $(\mathrm{P}<0,01)$. Este aspecto provavelmente está relacionado com à quantidade e às características dos sucedâneos fornecidos aos animais. Ainda considerando o PI, tudo indica que os bezerros levaram mais tempo para se adaptar ao sucedâneo do tratamento LAC. Por outro lado, no PII, o CMST dos bezerros do tratamento LAC não diferiu $(\mathrm{P}>0,05)$ do observado nos bezerros do tratamento LI. No tratamento DES, o CMST foi superior em 39,53 e 30,43\% em relação aos consumos observados nos bezerros dos tratamentos LAC e LI, respectivamente ( $\mathrm{P}<0,01)$. No PIII ( 0 a 60 dias) 
observou-se maior CMST nos bezerros do tratamento DES em relação ao consumo dos animais do tratamento LAC $(\mathrm{P}<0,01)$. A superioridade no CMST dos bezerros do tratamento DES em relação ao obtido no tratamento LAC foi de $38,23 \%$. Este fato é interessante, pois está relacionado com o melhor desempenho dos bezerros, inclusive com a possibilidade de torná-los ruminantes mais precocemente. Convém ressaltar que conforme observação de Garcia (1999), citado por Oliveira (2001), bezerros alimentados com sucedâneo durante o aleitamento, mesmo com desempenho pior ao do leite integral, conseguem recuperar-se pósdesmama.

Segundo o NRC (1989) o consumo diário de matéria seca de $1,33 \mathrm{~kg}$ é necessário para que bezerros da raça holandesa, com $50 \mathrm{~kg}$ de peso, ganhem $0,5 \mathrm{~kg} / \mathrm{dia}$, entretanto, na maioria dos trabalhos realizados no Brasil, o consumo de matéria seca por bezerros de $50 \mathrm{~kg}$ foi, em média, menor que um kg. Em experimentos com bezerros desaleitados próximo aos 60 dias de idade, verificou-se consumos de matéria seca total de $0,64 \mathrm{~kg} / \mathrm{dia}$ (VASCONCELOS, 1996); 0,86 kg/dia (SANDI \& MÜHLBACH, 1999); 0,74 kg/dia (VELHO et al., 2000) e $0,87 \mathrm{~kg} / \mathrm{dia}$ (NUSSIO et al., 2003).

Quanto às médias das mensurações corporais dos três tratamentos, verificou-se que apenas a CVe dos bezerros aos 28 e 42 dias de aleitamento foi influenciada pelos tratamentos, de tal forma que neste período os animais do tratamento LAC apresentaram menor CVe $(\mathrm{P}<0,05)$. Por outro lado, considerando-se o período total de aleitamento (60 dias), verificou-se que os tratamentos não influenciaram as mensurações corporais dos bezerros. Analisando-se a AC, pode-se notar que as médias foram muito próximas ( $\mathrm{P}>0,05)$, ou seja, 83,76; 81,91 e 81,81 cm nos tratamentos LI; DES e LAC, respectivamente. Os resultados foram semelhantes aos observados por Mattoso \& Garcia (1971), citados por Barreto (1993), 84,04 $\mathrm{cm}$, e superiores ao observado por Signoretti et al. (1995), $79,98 \mathrm{~cm}$. Analisando conjuntamente os dados das tabelas 4 e 6 verifica-se que no $42^{\circ}$ dia contido no subperíodo II, os animais do tratamento DES apresentaram maior crescimento do $\mathrm{CVe}$, o que atesta o maior desenvolvimento do rúmen destes animais já aos 42 dias, além do maior ganho em peso no subperíodo II, o que sugere que com o uso do sucedâneo Destetor ${ }^{\circledR} \mathrm{O}$ aleitamento poderia ser interrompido já aos 42 dias, ao invés dos 60 dias recomendados pelo fabricante. $\mathrm{Na}$ tabela 6 são apresentadas as médias de mensurações corporais dos animais aos 28,42 e 60 dias.

Considerando-se todas as características estudadas, os dois sucedâneos proporcionaram ganho em peso e desenvolvimento corporal satisfatórios durante o período de 60 dias, embora o tratamento com sucedâneo DESTETOR $^{\circledR}$ tenha proporcionado melhor desempenho aos animais já aos 42 dias. Além disto, o sucedâneo DESTETOR $^{\circledR}$ apresenta maior facilidade de preparo para o produtor por não exigir água quente, o que reduz o tempo de trabalho. O melhor desempenho dos bezerros do tratamento DES, possivelmente está relacionado com o melhor aproveitamento e aceitação do sucedâneo durante todo o período de aleitamento.

Tabela 5 - Médias de consumo de matéria seca, em kg/bezerro/dia, dos bezerros dos diferentes tratamentos e períodos experimentais, valores de F, erro padrão da média (EPM) e coeficientes de variação (CV).

\begin{tabular}{|c|c|c|c|c|c|c|c|c|c|c|c|c|}
\hline \multirow[b]{3}{*}{ Trat. } & \multicolumn{12}{|c|}{ Período (dias) } \\
\hline & \multicolumn{4}{|c|}{$I-(0-28)$} & \multicolumn{4}{|c|}{ II - $(29-56)$} & \multicolumn{4}{|c|}{ III - (0 a 60) } \\
\hline & $\mathrm{C}$ & $\mathrm{F}$ & $\mathrm{C}$ & $\mathrm{F}$ & $\mathrm{C}$ & $\mathrm{F}$ & $\mathrm{R}$ & $\mathrm{T}$ & $\mathrm{C}$ & $\mathrm{F}$ & $\mathrm{R}$ & $\mathrm{T}$ \\
\hline LI & 0,08 & 0,02 & 0,36 & 0,04 & 0,36 & 0,04 & 0,40 & $0,92 b$ & 0,24 & 0,02 & 0,27 & $0,79 \mathrm{ab}$ \\
\hline DES & 0,07 & 0,01 & 0,58 & 0,06 & 0,58 & 0,06 & 0,64 & $1,20^{\mathrm{a}}$ & 0,36 & 0,03 & 0,39 & $0,94^{\mathrm{a}}$ \\
\hline LAC & 0,07 & 0,00 & 0,43 & 0,05 & 0,43 & 0,05 & 0,48 & $0,86 b$ & 0,27 & 0,03 & 0,30 & $0,68 \mathrm{~b}$ \\
\hline Teste F & $0,24 \mathrm{~ns}$ & $2,14 \mathrm{~ns}$ & $2,59 \mathrm{~ns}$ & $0,43 \mathrm{~ns}$ & $2,59 \mathrm{~ns}$ & $0,43 \mathrm{~ns}$ & $2,64 \mathrm{~ns}$ & $5,97 * *$ & $1,80 \mathrm{~ns}$ & $0,29 \mathrm{~ns}$ & $1,82 \mathrm{~ns}$ & $6,87 * *$ \\
\hline EPM & 0,02 & 0,00 & 0,07 & 0,01 & 0,07 & 0,01 & 0,07 & 0,07 & 0,04 & 0,01 & 0,05 & 0,05 \\
\hline $\mathrm{CV} \%$ & 70,67 & 84,06 & 42,87 & 83,58 & 42,87 & 83,58 & 41,60 & 21,32 & 44,01 & 76,34 & 42,43 & 17,14 \\
\hline
\end{tabular}

$\mathrm{LI}=$ dieta contendo leite integral. $\mathrm{DES}=$ dieta contendo sucedâneo Destetor. $\mathrm{LAC}=$ dieta contendo sucedâneo Lactal. $\mathrm{F}=$ feno de capim-Tifton 85. $\mathrm{C}=$ concentrado. $\mathrm{R}=$ ração (concentrado + feno de capim-tifton 85 ). $\mathrm{T}=$ total (leite ou sucedâneo + ração). $\mathrm{ns}$ $=$ não significativo $(\mathrm{P}>0,05) . * *(\mathrm{P}<0,01)$. Médias seguidas de letras iguais na coluna, não diferem entre si, pelo teste de Tukey. 
Tabela 6 - Médias da altura na cernelha (AC), da circunferência torácica (CT) e da circunferência ventral (CVe), dados em cm, dos bezerros dos diferentes tratamentos, valores do Teste F, Erro padrão da média (EPM) e coeficientes de variação $(\mathrm{CV})$.

\begin{tabular}{|c|c|c|c|c|c|c|c|c|c|}
\hline \multirow[b]{2}{*}{ Trat/dias } & \multicolumn{3}{|c|}{$\mathrm{AC}$} & \multicolumn{3}{|c|}{ CT } & \multicolumn{3}{|c|}{$\mathrm{CVe}$} \\
\hline & 28 & 42 & 60 & 28 & 42 & 60 & 28 & 42 & 60 \\
\hline LI & 79,64 & 81,01 & 83,76 & 83,27 & 85,77 & 89,14 & $82,39^{a}$ & $83,89^{a}$ & 89,02 \\
\hline DES & 78,51 & 79,89 & 81,91 & 82,39 & 86,38 & 91,99 & $81,83^{\mathrm{a}}$ & $86,08^{\mathrm{a}}$ & 92,18 \\
\hline LAC & 78,71 & 79,59 & 81,81 & 79,83 & 83,46 & 87,72 & $76,14^{\mathrm{b}}$ & $78,40^{\mathrm{b}}$ & 85,04 \\
\hline Teste F & $0,85^{\mathrm{ns}}$ & $0,36^{\mathrm{ns}}$ & $1,81^{\mathrm{ns}}$ & $2,66^{\mathrm{ns}}$ & $1,74^{\mathrm{ns}}$ & $3,38^{\mathrm{ns}}$ & $4,10^{*}$ & $4,37^{*}$ & $3,33^{\mathrm{n}}$ \\
\hline EPM & 0,652 & 0,732 & 0,592 & 1,090 & 1,156 & 1,169 & 1,696 & 1,874 & 1,936 \\
\hline $\mathrm{CV}, \%$ & 2,33 & 2,57 & 2,79 & 3,75 & 3,82 & 3,68 & 5,97 & 6,38 & 6,15 \\
\hline
\end{tabular}

Médias seguidas de letras iguais, não diferem entre si, pelo teste de Tukey $(\mathrm{P}>0,05)$. LI = dieta contendo leite integral. DES $=$ dieta contendo sucedâneo Destetor. $\mathrm{LAC}=$ dieta contendo sucedâneo Lactal. EPM = Erro Padrão da Média. $\mathrm{CV}=\mathrm{Coeficiente} \mathrm{de}$ Variação. * $(\mathrm{P}<0,05) . \mathrm{ns}=$ não significativo $(\mathrm{P}>0,05)$.

\section{CONCLUSÕES}

A utilização tanto do sucedâneo DESTETOR ${ }^{\circledR}$ quanto do LACTAL $^{\circledR}$ foi viável tecnicamente, proporcionando aos animais desempenhos satisfatórios, em comparação ao leite integral, durante o período total de aleitamento de bezerros.

\section{REFERÊNCIAS BIBLIOGRÁFICAS}

BANZATTO, D. A.; KRONKA, S. N. Experimentação agrícola. Jaboticabal: FUNEP, 1992. 247 p.

BARBOSA, J. C.; MALHEIROS, E. B.; BANZATTO, D. A. ESTAT: um sistema de análises estatísticas de ensaios agronômicos. Versão 2.0. Jaboticabal: Unesp, 1992.

BARRETO, L. C. N. Utilização de misturas de "leite" de soja e soro de queijo no aleitamento de bezerros. 1993. 51 f. Dissertação (Mestrado em Zootecnia) - Universidade Federal de Lavras, Lavras, 1993.

CAMPOS, O. F. de; LIZIEIRE, R. S. Alimentação de bovinos jovens. Belo Horizonte: UFMG, 1995. (Cadernos técnicos, 14)

GERMANO, J. L. Utilização de substitutos de leite a base de soja e soro de queijo na alimentação de bezerros. 1992. 91 f. Dissertação (Mestrado em Zootecnia) - Universidade Federal de Lavras, Lavras, 1992.

JAURIS, G. C.; SANCHEZ, L. M. B.; VIEGAS, J.; VELHO, J. P.; CARVALHO, P. A.; RODRIGUES, M. B. Efeito da substituição parcial de leite por dieta sólida no desaleitamento precoce de bezerros holandeses. In:
REUNIÃO ANUAL DA SOCIEDADE BRASILEIRA DE ZOOTECNIA, 36., 1999, Porto Alegre. Anais... Porto Alegre: SBZ, 1999. CD-ROM.

LOMBARDI, C. T.; CASTRO, A. C. G.; SILVA, J. F. C.; PEREIRA, J. C.; VELHO FILHO, S. C.; CECON, P. R. Desempenho de bezerros desaleitados precocemente submetidos a restrição no fornecimento de leite. In: REUNIÃO ANUAL DA SOCIEDADE BRASILEIRA DE ZOOTECNIA, 34., 1997, Juiz de Fora. Anais... Juiz de Fora: SBZ, 1997.

LOPES, J. N. P.; CAMPOS, O. F.; LEÃO, M. I. Efeito de dietas líquidas à base de leite integral e/ou, subprodutos de soja sobre algumas características relacionadas à digestão, em bezerros. Revista da Sociedade Brasileira de Zootecnia, Viçosa, v. 27, n. 3, p. 603-612, mar. 1998.

LUCCI, C. S. Bovinos leiteiros jovens: nutrição, manejo, doenças. São Paulo: Nobel; Edusp, 1989. 371 p.

McDOWELL, L. R.; CONRAD, J. H.; THOMAS, J. E.; HARRIS, L. E. Tabelas de composição de alimentos para a América Latina. Gainesville: Universidade da Flórida, 1974. $47 \mathrm{p}$.

NATIONAL RESEARCH COUNCIL. National requirements of dairy cattle. Washington, DC, 1989. 157 p.

NUSSIO, C. M. B.; RODRIGUES, A. A. Avaliação de critérios para desaleitamento de bezerras leiteiras. In: REUNIÃO ANUAL DA SOCIEDADE BRASILEIRA DE ZOOTECNIA, 40., 2003, Santa Maria. Anais... Santa Maria: SBZ, 2003. CD-ROM. 
OLIVEIRA, M. D. S. Cria e recria de bovinos leiteiros. Jaboticabal: FUNEP, 2001. 180 p.

PLAZA, J.; FERNANDEZ, J. L. Artificial rearing of calves in dairy farms. Cuban Journal of Agricultural Science, Havana, v. 31, p. 21-23, 1997.

SANDI, D.; MÜHLBACH, R. F. Desempenho de bezerros holandês com desaleitamento aos 28 e ou 56 dias de idade, com ou sem aditivo a base de oligossacarídeo de manana. In: REUNIÃO ANUAL DA SOCIEDADE BRASILEIRA DE ZOOTECNIA, 36., 1999, Porto Alegre. Anais... Porto Alegre: SBZ, 1999. CD-ROM.

SIGNORETTI, R. D.; CASTRO, A. C. G.; SILVA, J. F. C. Utilização do farelo de gérmen de milho no concentrado inicial de bezerros de raças leiteiras em sistemas de desaleitamento precoce. Revista da Sociedade Brasileira de Zootecnia, Viçosa, v. 24, n. 5, p. 847-851, maio 1995.

SUSIN, I.; MORAES NETO, R.; PIRES, A. V.; PACKER, I. U. Desempenho de bezerros submetidos a dietas líquidas e períodos de aleitamento. Revista da Sociedade Brasileira de Zootecnia, Viçosa, v. 17, n. 1, p. 108-114, jan. 1988.

VASCONCELOS, M. A. Desempenho de bezerros da raça holandesa nascidos durante o período outono/inverno submetidos a diferentes dietas líquidas e instalações. 1996. 81 f. Dissertação (Mestrado em Zootecnia) - Universidade Federal de Santa Maria, Santa Maria, 1996.

VELHO, J. P.; VIÉGAS, J.; SILVA, J. H. S.; RODRIGUES, M. B.; BURIN, R.; RAMOS, R. C. Quantificação das necessidades diárias de leite para bezerros holandeses até dois meses de idade. In: REUNIÃO ANUAL DA SOCIEDADE BRASILEIRA DE ZOOTECNIA, 37., 2000, Viçosa. Anais... Viçosa: SBZ, 2000. CD-ROM.

ZANETTI, M. A.; SCHALCH, E.; ROSSINI, A. J.; SARAN NETO, A.; OLIVEIRA, M. G.; MORGONNI, D. C. Uso de aditivos em dietas de bezerros holandeses. In: REUNIÃO ANUAL DA SOCIEDADE BRASILEIRA DE ZOOTECNIA, 36., 1999, Porto Alegre. Anais... Porto Alegre: SBZ, 1999. CD-ROM. 